ISSN 1991-8631

Original Paper

http://indexmedicus.afro.who.int

\title{
Étude de l'œstrus et de la fertilité après un traitement de maîtrise des cycles chez les femelles zébus
}

\author{
Wéré PITALA ${ }^{1,5^{*}}$, Moussa ZONGO ${ }^{2}$, Hamidou BOLY ${ }^{3}$, Laya SAWADOGO ${ }^{2}$, \\ Pascal LEROY ${ }^{4}$, Jean- François BECKERS ${ }^{4}$ et Messanvi GBEASSOR ${ }^{5}$ \\ ${ }^{I}$ Ecole Supérieure d'Agronomie, Université de Lomé, BP 1515 Lomé - Togo. \\ ${ }^{2}$ UFR/SVT, Université de Ouagadougou, 03 BP 7021 Ouagadougou 03 - Burkina Faso. \\ ${ }^{3}$ Institut du Développement Rural, Université Polytechnique de Bobo-dioulasso, 01 B.P. 1091 Bobo-Dioulasso \\ 01 - Burkina Faso. \\ ${ }^{4}$ Faculté de Médecine Vétérinaire. Université de, Liège, Bd. de Colonster, 20, B43-4000 Sart tilman, \\ Liège - Belgique. \\ ${ }^{5}$ Faculté des Sciences, Université de Lomé, BP 1515 Lomé, Togo. \\ * Correspondant : Ecole Supérieure d'Agronomie, Université de Lomé, BP 1515 Lomé - Togo \\ Tel :002289094027.E-mail : werepit@hotmail.com
}

\section{RESUME}

La recherche a visé à établir les taux de réponse et conception des animaux synchronisés au Crestar® associé à la PMSG. 39 femelles zébus (Bos indicus) Goudali et Azawak constitué de vaches et de génisses ont été utilisées dans l'étude. L'insémination artificielle a été faite de façon systématique 48 heures après l'arrêt du traitement. Le diagnostic de gestation a été déterminé 60 jours après insémination par palpation rectale. Le taux global de l'œstrus induit a été de 94,87\%. La parité et la race de femelles n'ont eu aucun effet significatif sur la réponse de l'œstrus. Le délai d'apparition de l'œstrus a été de 30,11 $\pm 6,24$ heures et 32,76 \pm 4,75 heures après la fin du traitement respectivement pour les femelles zébus Goudali et Azawak. La durée moyenne de l'œstrus a été de $12,20 \pm 1,30$ heures $(n=5)$. Le taux global de fertilité a été de $41,03 \%$. La parité et la race de femelles n'ont eu aucun effet significatif sur la fertilité.

() 2012 International Formulae Group. All rights reserved.

Mots clés : Insémination artificielle, Crestar, taux de gestation, synchronisation, Niger.

\section{INTRODUCTION}

Depuis quelques années, les chercheurs de la zone tropicale et sub-tropicale ont tenté par différents moyens d'augmenter l'efficacité des troupeaux bovins en vue de couvrir les besoins en lait et produits laitiers d'une population dont le taux de croissance est l'un des plus élevés du monde. Toutefois, les facteurs limitants sont nombreux. Parmi les plus importants, il faut noter la quasigénéralisation de l'utilisation de l'insémination artificielle dans les troupeaux permettant d'accélérer le progrès génétique. La détection des chaleurs, consomatrice de temps pourrait s'avérer difficile avec des conséquences sur les résultats de reproduction ou devenir un frein de l'insémination artificielle (Boly et al., 2003 ; Seegers et al., 
2010). Or dans les élevages de la zone tropicale et sub-tropicale, la détection de l'œstrus est très difficile pour les bouviers qui n'ont aucune formation dans le domaine d'une part et d'autre part, les races bovines de cette zone présentent des chaleurs naturelles de courte durée et souvent discrètes (Mattoni et al., 1988 ; Chicoteau, 1991).

Afin d'améliorer la détection de l'œstrus, plusieurs protocoles de maîtrise de cycle, ayant pour objet de synchroniser l'œstrus et l'ovulation ont été proposés chez les taurins d'Europe et d' Amérique. Les plus répandus utilisent soient les progestagènes (ou leurs analogues), soient les prostaglandines (ou leurs analogues) associées ou non à la PMSG (Pregnant Mare Serum Gonadotropin) (ou leurs analogues) selon plusieurs schémas donnant des résultats satisfaisant de fertilité après insémination artificielle (Cecyre et al., 2001 ; Dezaux, 2001 ; Beffera, 2007).

Cependant, les résultats de quelques expériences réalisées en Afrique soudanosahélienne sur les zébus montrent un taux de fertilité relativement faible (Zongo et al., 2001 ; Pitala et al., 2005 ; Marichatou et al., 2010).

L'objectif de notre étude consiste à évaluer l'efficacité d'une méthode d'induction et de synchronisation de l'œstrus chez les femelles zébus Goudali et Azawak, associant les progestagènes et la PMSG dans une ferme semi-intensive au Niger.

\section{MATERIEL ET METHODES}

\section{Zone expérimentale}

La présente étude a été menée dans un élevage semi-intensif, situé à $50 \mathrm{~km}$ à l'est de Niamey au Niger. Cette zone d'étude jouit d'un climat de type sud-sahélien, caractérisé par une saison sèche d'octobre à mai (période d'expérience) et une saison des pluies de juin à septembre. Les précipitations varient de 400 $\mathrm{mm}$ à $600 \mathrm{~mm}$ de pluies par an. Les températures varient de $15{ }^{\circ} \mathrm{C}$ en janvier à 41 ${ }^{\circ} \mathrm{C}$ en avril.

\section{Animaux}

Trente neuf (39) femelles zébus (Bos indicus) ayant les caractéristiques suivantes ont été concernées. Elles étaient âgées de 2,5 ans à 5 ans. Elles présentent un état corporel acceptable au début du traitement hormonal. Ces femelles ont été normalement déparasitées et vaccinées.

\section{Induction d'œstrus}

Toutes les femelles ont subi un diagnostic de non gestation par palpation rectale avant d'être soumises au protocole d'induction et de synchronisation de l'œstrus associant les progestagènes et la PMSG selon le protocole suivant :

- jour 0: Pose de l'implant Creastar ${ }^{\circledR}$, Intervet (3 $\mathrm{mg}$ de Norgestomet, Intervet, France) par voie sous cutanée sous le pavillon de l'oreille et injection intramusculaire de $2 \mathrm{ml}$ de solution huileuse de Valérate d'oestradiol, Intervet $(3,8 \mathrm{mg})$;

- jour 10: Retrait de l'implant et injection intramusculaire de 400 UI de Pregnant Mare Serum Gonadotropin (PMSG) (Folligon®, Intervet, France) correspondant à la fin du traitement.

\section{Insémination artificielle et évaluation de la fertilité}

Pour des raisons pratiques, les inséminations ont été réalisées à la $48^{\text {ème }}$ heure après la fin du traitement. Les inséminations ont été pratiquées avec la semence congelée des taureaux 'Holstein' dont la fertilité est réputée bonne (Linalux, Ciney, Belgique) avec un taux de réactivité de $80 \%$.

\section{Paramètres étudiés}

La détection de l'œstrus est réalisée de façon continue par observations visuelles continues des modifications comportementales et les signes cliniques de l'œstrus obtenus par les palper trans-rectal après le retrait des implants pendant 48 
heures. Les critères majeurs de l'œstrus retenus sont l'acceptation du chevauchement et l'immobilisation de la femelle lorsqu'elle est chevauchée par une autre femelle du groupe, la présence de glaires, la perméabilité du col de l'utérus (Ponsart et Humblot, 2002).

Les paramètres étudiés ont été le délai d'apparition et la durée de l'œstrus. Le délai d'apparition de l'œstrus a été défini comme le temps s'écoulant entre le retrait de l'implant et l'observation des premiers chevauchements acceptés. Le début de l'œstrus a été défini comme le moment du premier chevauchement observé. La fin de l'œstrus a été définie comme le moment du dernier chevauchement observé, sans que d'autres chevauchements ne soient observés dans les 12 heures suivantes (White et Wettermann, 2000). La durée moyenne de l'œstrus a été calculée chez cinq (5) femelles sans distinction de la race et de la parité.

Le diagnostic de gestation a été effectué par palpation rectale 60 jours après l'insémination artificielle. Toutes les gestations ont été confirmées par les misebas.

\section{Analyse statistique}

Les résultats ont été exprimés en moyenne \pm écart type et les différences ont été considérées comme significatives au seuil de probabilité de $\mathrm{P} \leq 0,05$.

Par ailleurs, l'effet du traitement sur les différentes variables (œstrus, délai d'apparition de l'œstrus et les taux de fertilité) a été apprécié à l'aide du programme ANOVA.

\section{RESULTATS \\ Induction de l'ostrus}

Sur l'effectif de 39 femelles, seules 2 femelles ne sont pas venues en chaleurs, soit un taux global de synchronisation de $94,87 \%$ 48 heures après le retrait de l'implant suivi de l'injection de la PMSG. Le Tableau 1 représente la répartition du taux de l'œstrus selon la race et la parité. La parité et la race des femelles n'ont eu aucun effet significatif sur la réponse de l'œstrus.

Le délai moyen d'apparition de l'œstrus est de 32,76 $\pm 4,75$ heures et 30,11 \pm 6,24 heures avec des variations allant de la $23^{\text {ème }}$ heure (zébu Azawak) à la $43^{\text {ème }}$ heure (zébu Goudali) après la fin du traitement.

La durée moyenne de l'œstrus est de $12,2 \pm 1,30$ heures avec des valeurs extrêmes de 11 heures et 14 heures.

\section{Fertilité en insémination artificielle}

Le taux global de fertilité à l'œstrus induit par les implants Crestar® associé à la PMSG est $41,03 \%$. Ce taux varie suivant la race et la parité des femelles (Tableau 1). Mais ces variations n'ont aucun effet significatif sur la fertilité selon nos analyses statistiques.

Tableau 1 : œstrus induit et fertilité des femelles zébus traitées au implant Crestar® associé à la PMSG.

\begin{tabular}{|c|c|c|c|c|}
\hline Facteurs & & Effectif & Estrus & Gestation \\
\hline \multirow[b]{2}{*}{ Parité } & Vaches & 18 & $94,44 \%(17 / 18)$ & $44,44 \%(8 / 18)$ \\
\hline & Génisses & 21 & $95,24 \%(20 / 21)$ & $38,10 \%(8 / 21)$ \\
\hline \multirow{3}{*}{ Race } & Goudali & 20 & $95 \%(19 / 20)$ & $45 \%(9 / 20)$ \\
\hline & Azawak & 19 & $94,74 \%(17 / 19)$ & $36,84 \%(7 / 19)$ \\
\hline & Total & 39 & $94,87 \%$ & $41,03 \%$ \\
\hline
\end{tabular}




\section{DISCUSSION}

La synchronisation de l'œstrus est permise par l'utilisation de plusieurs méthodes. Une des méthodes de synchronisation de l'œstrus se fait par deux injections de prostaglandines, mais uniquement sur les femelles cyclées. Cette méthode est moins utilisée en Afrique du fait du faible taux de femelles cyclées dans les élevages en Afrique Soudano-sahélienne. D'autres méthodes permettent à la fois d'induire les chaleurs et de les synchroniser. Ces méthodes sont utilisables sur des vaches cyclées et non cyclées mais pubères. Les deux méthodes les plus utilisées pour induire et synchroniser l'œstrus sont la spirale vaginale et d'implant sous-cutané.

Le taux moyen de synchronisation de l'œstrus $(94,87 \%)$ est conforme aux résultats de Mbaye (1990) (92,8\%) avec implant Crestar ${ }^{\circledR}$ associé à la PMSG, et Ndiaye (1992) (93,8\%) avec Crestar® seul au Sénégal. Par contre, il est inférieur à ceux de Ouedraogo et al. (1996) qui ont rapporté un taux de $100 \%$ chez les zébus à la station expérimentale du C.I.R.D.E.S. (Centre International de Recherche-Développement sur l'Elevage en zone Subhumide), à Banankélédaga dans le sud ouest du Burkina Faso et de Pitala et al. (2005) (96,9\%) avec le Crestar ${ }^{\circledR}$ associé à la prostaglandine et à la PMSG au Burkina Faso chez les zébus Goudali. Mais ce taux est supérieur à celui obtenu par Messine et al. (1993) (42,5\%) avec l'implant sous-cutané seul à l'institut de recherche zootechnique de Wakwa au Cameroun.

Le délai moyen d'apparition des chaleurs, observé chez les femelles zébu Goudali et Azawak au cours de cette étude est similaire à celui $(32,0 \pm 1,1$ heures $)$ rapporté par Tegegne et al. (1989) chez les femelles zébu Boran. Par contre, ce délai est proche de $34,0 \pm 1,5$ heures observé par Twagiramungu et al. (1993) à l'issue d'une synchronisation par les implants Crestar® et nettement inférieur à celui (51 heures 40 minutes) rapporté par Ouedraogo et al. (1996).
La durée moyenne de l'œstrus chez les femelles zébus Goudali et Azawak soumises au protocole d'induction et de synchronisation de l'œstrus dans cette étude se situe dans les valeurs limites rapportées par Voh et al. (1987) chez les vaches zébus Bunaji et Goudali pendant la saison sèche. Cette durée moyenne de l'œstrus est proche de celle de 14,9 \pm 0,6 heures observée chez les génisses zébus Hariana et Sachiwal par Singh et al. (1998). Mais, elle est cependant supérieure à celle rapportée par Johnson et Oni (1986) chez Bunaji cross au Nigeria (8,2 $\pm 2,8$ heures); par Mattoni et al. (1988) chez les femelles Small East African Zebu en Ethiopie (2,5 à 7,4 heures). Le taux moyen de fertilité chez les femelles zébu Goudali et Azawak après œstrus induit par les implants Crestar ${ }^{\circledR}$ associés à la PMSG s'inscrit dans les limites (40-50\%) rapportées par Mbaye et al. (1989); par Mbaye et Ndiaye (1993) chez le zébu Cobra au Sénégal; par Pitala et al. (2005) sur les femelles zébus Goudali au Burkina et par Tada et al., (2010) sur les zébus au Zimbabwe. Par contre ces résultats sont supérieurs à ceux obtenus par Diadhiou, 2001 (27,7\%) avec Crestar® chez le zébu Cobra au Sénégal. Il faut noter que le même auteur a obtenu un taux de fertilité de $42,7 \%$ avec le Prid® chez la même race de zébu (Cobra). Mais, les différences ne vont pas toutes dans le même sens et dans tous les élevages. Ceci est probablement dû aux caractéristiques des troupeaux mis à l'expérimentation et aux semences probablement comme l'a démontré Data et al. (2010) au Zimbabwe. Le taux de gestation est aussi très dépendant de l'état physiologique de l'animal avant le traitement et de la saison de l'expérience (Zongo et al., 2011). Selon Freret et al. (2011), la parité affecte la fertilité chez les vaches Prim'Holstein, le taux de gestation étant plus élevé chez les primipares. Tous ces facteurs intervenant dans la variation du taux de gestation ont été montrés en long et en large par Bouyer (2006) à travers divers projets d'insémination artificielle en Afrique soudano-sahélienne. 


\section{Conclusion}

Le traitement à base d'implants Crestar ${ }^{\circledR}$ associés à la PMSG permet une bonne induction et/ou synchronisation de l'œstrus chez les femelles zébus Goudali et Azawak, avec des taux de $95 \%$ et $94,74 \%$ respectivement et un taux global de $94,87 \%$ dans les 48 heures qui suivent l'arrêt du traitement.

Avec un taux moyen de gestation de $41,03 \%$ avec des variations de $45 \%$ et $36,84 \%$ respectivement chez les femelles zébus Goudali et Azawak à l'insémination artificielle à 48 heures après le retrait de l'implant suivi de l'injection intramusculaire de la PMSG, nous pouvons dire que cette méthode ne pose pas de problèmes de fertilité.

Cependant, le faible effectif des femelles utilisé ne nous permet pas de tirer une conclusion définitive. Mais, les résultats montrent que ce protocole permet de résoudre le problème de détection de l'œstrus dans les élevages de la zone tropicale et sub-tropicale chez les femelles zébus Goudali et Azawak. Ceci ouvre une voie dans les programmes d'application de la première génération des biotechnologies de la reproduction en vue d'une amélioration du progrès génétique en élevage en Afrique sub-sahélienne.

\section{REMERCIEMENTS}

Les auteurs de cet article tiennent à remercier le projet CIUF/U.O./SPA de IUFR/SVT de l'Université de Ouagadougou au Burkina Faso pour son soutien financier dans l'achat des produits pharmaceutiques de synchronisation, le Lynalux pour la fourniture des semences et le laboratoire de physiologie de la reproduction de la Faculté de Médecine Vétérinaire de l'Université de Liège en Belgique pour son soutien logistique dans la réalisation de cette étude.

\section{REFERENCES}

Beffera C. 2007. Comparaison de l'efficacité $\mathrm{du}$ traitement de synchronisation des chaleurs Crestar® classique et d'un nouveau traitement combinant buséréline implant Crestar®, prostaglandine $\mathrm{F} 2 \alpha$ et eCG chez la vache allaitante. Thèse de doctorat de médecine vétérinaire, Ecole Nationale Vétérinaire d'Alfort, p. 100.

Boly H, Coulibaly I, Pitala W, Ba Y, Sawadogo L. 2003. Comportement sexuel et maturation folliculaire chez la femelle zébu Goudali au Burkina Faso. Revue Africaine de Santé et des Productions Animales, 1: 102-107.

Bouyer B. 2006. Bilan et analyse de l'utilisation de l'insémination artificielle dans les programmes d'amélioration génétique des races laitières en Afrique soudano-sahélienne. Thèse de Doctorat de Médecine Vétérinaire, Ecole Nationale Vétérinaire de Lyon, Lyon, p. 108.

Cecyre D, Descoteaux L, Bouchard E. 2001. Evaluation d'un protocole de synchronisation de l'ovulation, avec l'insémination artificielle sur rendezvous, utilisant la GnRH et la PgF2 $\alpha$ chez la vache laitière. Le Médecin Vétérinaire du Québec, 31: 130-133.

Chicoteau P. 1991. La reproduction des bovins tropicaux. Recueil de Médecine Vétérinaire Spécial Reproduction des Ruminants (numéro spécial): 241-246.

Dezaux P. 2001. Synchronisation des chaleurs chez les vaches allaitantes par association GnRH-PgF2 $\alpha-$ GnRH. Thèse de doctorat de médecine vétérinaire, Ecole Nationale Vétérinaire d'Alfort, p. 91.

Diadhiou A. 2001. Etude comparative de deux moyens de maîtrise de la reproduction (L'implant CRESTAR® et la Spirale PRID®) chez les vaches N'Dama et Cobra au Sénégal. Thèse de Doctorat de Médecine Vétérinaire, EISMV, Dakar, p. 84.

Freret S, Salvetti P, Gatien J, Humblot P, Ponsart C. 2011. Facteurs de variation de la fertilité en première insémination artificielle chez les vaches Prim'Holstein. 
Rencontres Recherches Ruminants, 18: 89-92.

Johnson AO, Oni OO. 1986. Estrus detection by mounts received in Friesian $\times$ Bunaji and Bunaji heifers. Journal of Agricultural Science, 107: 67-69.

Marichatou H, Issa M, Hamadou I, Assane M, Semita C. 2010. Efficacité de la synchronisation des chaleurs et l'insémination artificielle chez les bovins Azawak: intérêt du profil de progestérone. Tropicultura, 28: 161-167.

Mattoni M, Mukasa-Mugerwa E, Gecchini G, Savoni S. 1988. The reproductive performance of East Africa (Bos indicus) zebu cattle in Ethiopia: Estrus cycle lenth, duration, behavior and ovulation time. Theriogenology, 30: 961-971.

Mbaye M, Ndiaye M. 1993. Etude des chaleurs et de la fertilité après un traitement de maîtrise de la reproduction chez la vache zébu Cobra. Actualité Scientifique, 27-37.

Mbaye M, Diop PEH, Ndiaye M. 1989. Etude du cycle sexuel chez la vache race N'Dama. In Atelier Reprod. Bétail Trypanotolérant. Afrique (FAORAF/88/100) Banjul, 34-35.

Mbaye M. 1990. Induction et synchronisation des chaleurs chez la femelle zébu Cobra. CRZ de DAHRA. Rapport de confirmation, ISRA-LNERV.

Messine O, Mbah D, Saint Martin G. 1993. Synchronisation de l'œstrus chez les femelles zébu Goudali au CRZ de Wakwa (13-20). In Maîtrise de la Reproduction et Amélioration Génétique des Ruminants. Actualité Scientifique AUPELF-UREF.

Ndiaye M. 1992. La diffusion du progrès génétique par la mise en place de géniteurs ou par l'IA au Sénégal. In L'Amélioration Génétique des Bovins en Afrique de l'Ouest. FAO: Rome ; 283- 289.
Ouedraogo A, Mattoni M, Zecchini M. 1996. Définition d'un moment optimum pour l'insémination artificielle chez les femelles bovines Baoulé, Zébu et N'dama en zone subhumide. Reproduction et Production Laitière, 305-310.

Pitala W, Zongo M, Boly H, Coulibaly I, Ba Y, Sousa NM, Leroy P, Beckers JF, Sawadogo L. 2005. Induction des chaleurs chez le zébu Goudali : observation de l'œstrus et insémination artificielle. Annales de l'Université de Ouagadougou, série C : 117-139.

Ponsart C, Humblot P. 2002. Détection des Chaleurs chez les Bovins. Gestation. Societé Française de Buiatrie.

Seegers H, Billon D, Bossard-Appere E, Ponsart C, Paccard P, Disenhaus C. 2010. Evaluation retrospective de la qualité de la detection des chaleurs en troupeaux bovin laitier à partir des données déjà disponibles. Rencontre Recherche Ruminants, 17: 133-136.

Singh U, Khurama NK, Inderjee T. 1998. Plasma progesterone profiles and fertility status of anestrus zebu cattle treated with norgestomet-estrdiol-eCG regimen. Theriogenology, 50: 1191-1199.

Tada O, Masamba B, Gadzirayi CT. 2010. Efficacy of Crestar (progesterone analogue) and prosolvin (prostaglandin analogue) in heat synchronization of indigenous small holder dairy and commercial beef cows. Electronic Journal of Environnemntal Agroculture and Food Chemistry, 385-395.

Tegegne A, Warnick AC, Musaka-Mugerwa E, Ketema H. 1989. Fertility of Bos indicus and Bos indicus $\mathrm{x}$ Bos taurus crossbreed cattle after estrus synchronization. Theriogenology, 31: 361-370.

Twagiramungu H, Guibault LA, Villeneuve P, Proulx J, Dufour JJ. 1993. Récents Développements dans la Synchronisation de l'œstrus et la Fertilité en Insémination 
Artificielle Bovine. In Actualité scientifique: Maîtrise de la Reproduction et Amélioration Génétique des Ruminants. Apports des Technologies Nouvelles. Les Nouvelles Editions du Sénégal ; 39-56.

Voh AA, Oyedipe EO, Buvanendran V, Kumi-Diaka J. 1987. Estrus response of indigenous Nigeria Zebu cows after prostaglandin $\mathrm{F}_{2}$ alpha analogue treatment under continuous observations for two seasons. Theriogenology, 28: 77-99.

White FJ, Wettermann RP. 2000. Season alters estrus behavior but not time of ovulation in beefs cows. Animal Science Research Report; 1-6.

Zongo M, Boly H, Sawadogo L, Pitala W, Sousa NM, Beckers JF, Leroy P. 2001. Insémination artificielle des vaches 'Azawak' et taurin 'Gourunsi' au Burkina Faso. Tropicultura, 19: 75-78.

Zongo M, Pitala W, Sawadogo L, Boly H, Beckers JF. 2011. Efficacité d'un traitement de maîtrise des cycles chez les zébus: œstrus induit et fertilité. Journal Recherche Scientifique Université de Lomé (sous presse). 